\title{
Modelling in Ecology, Epidemiology and Ecoepidemiology: Introduction to the Special Issue
}

\author{
A. Morozov $^{1,2}$, S. Petrovskii ${ }^{1}$ \\ ${ }^{1}$ Department of Mathematics, University of Leicester, Leicester, LE1 7RH, UK \\ ${ }^{2}$ Shirshov Institute of Oceanology, Moscow, 117997, Russia
}

\begin{abstract}
Keywords and phrases: bifurcation theory, sensitivity, food-web models, model reduction, spatial ecology
\end{abstract}

Mathematics Subject Classification: 92B, 92D, 34C, 92D25, 37N25, 34H, 49K

Mathematical modelling in ecology, epidemiology and eco-epidemiology has long been recognized as a powerful research tool [14]. Indeed, mathematical models create a convenient virtual environment where various hypotheses can be checked and different scenarios studied in much detail. Mathematical modelling and simulations in ecology therefore provide a convenient and efficient supplement, and sometimes even an alternative, to field experiments that are costly and often difficult to replicate because of the inherently transient nature of environmental conditions. In epidemiology, the role of mathematical modelling is perhaps even more important as experimenting with dangerous diseases is hardly possible at all.

The main focus of this Special Issue is on the following areas of theoretical ecology, epidemiology and biological evolution: (i) modelling the role of spatial heterogeneity and dispersal in species persistence; (ii) new methods of sensitivity analysis and parameters estimation in biological models; (iii) advances in modelling of infectious diseases; (iv) reduction of complex ecological models to simpler tractable models and (v) evolution of dispersal. It is worth emphasizing that most of the contributions to the Issue are not restricted to only one of the mentioned topics, but combine few of them, so it may be hard to assign a particular article to a single topic.

In mathematical ecology, it is well recognized the importance of spatial heterogeneity for population establishment andd/or persistence [8,10,12]. The contribution of Alharbi and Petrovskii [2] explores the effects of the size and the shape (geometry) of the habitat on the persistence of a single population subject to the Allee effect. The authors consider the possibility of population survival in several types of the habitat, in particular, in rectangular, cross-shaped and $\mathrm{H}$-shaped habitats and across various sizes of the domain. Extensive simulations have been completed for a wide range of parameters, characterizing viability of population, in particular, the Allee threshold value. It is convincingly demonstrated that both size and the geometry of the habitat have crucial effect on population survival and persistence. Interestingly, it is observed that species that maintains its maximum growth rate would likely have more chances to survive in a complex or heavily fragmented environment than species maintaining its maximum per capita growth rate, which apparently points out at a certain evolutionary strategy.

(C) EDP Sciences, 2016 
In their study, Egilmez and Morozov [5] revisit the role of environmental heterogeneity, food web structure and shape of the predator functional response in efficiency of top-down control in eutrophic ecosystems. As a case study, they consider a vertically resolved tri-trophic planktonic food web composed of a primary producer, an intermediate predator and a highly mobile top predator. It was shown that the interplay between environmental heterogeneity (due to light attenuation) and trophic interaction can result in a strong top-down control which would otherwise be impossible in the corresponding well-mixed system. The demonstrated mechanism of top-down control seems to be rather generic and might as well be a good candidate to explain stability in some non-planktonic tri-trophic ecosystems where other mechanisms either do not apply or fail to stabilize the system.

In his contribution, H. Seno [24] revisits the metapopulation model of Levins' type, which has long remained a central paradigm in the ecological literature. Namely, the author re-considers a 3-state metapopulation dynamics model of Hanski [6] and the original two-species model by Levins [11]. H. Seno [24] introduces an explicit dispersal compartment and makes use of the difference in time scales between the state transition and the dispersal of individuals across the habitat. It is shown in the paper that the original metapopulation model of Levins' type (based on mass-action terms) are valid only under some additional implicit assumptions that do not necessarily hold in realistic situations.

The papers by ten Broeke et al. [23] and Tyukin et al. [25] explore the sensitivity of biological models to the choice of parameters as well as the reliable ways of parameter estimations, which is a fundamental issue in mathematical biology $[1,26]$. In their work, ten Broeke and co-authors compare different methodologies for sensitivity analysis of ecological models accounting for the existence of tipping points. The main claim is that when in our sensitivity analysis we do not consider tipping points, this may result in misleading statistics on model behaviour. ten Broeke et al. explore how different methodologies of sensitivity analysis work for models with tipping points by considering a classical predator-prey model. They conclude that the occurrence of tipping points can be found only from combination of different sensitivity analysis methodologies. Tyukin and co-authors [25] have developed a new method for efficient data-based parameter estimation of ODE-based models in the case where the solutions are periodical. Although the method is applicable for a particular type of models, the class of the models is rather broad, in particular, it is applicable to various predator-prey systems and models in physiology. Technically, the method is based on representation of a periodic solution as a sum of computable integrals and functions which explicitly depends on unknown parameters. This allows implementation of parallel computing, which highly increases the performance of the method. The method is efficiently applied to a predatorprey system and Morris-Lecar model for oscillations in barnacle giant muscle fiber [15].

The works of Grigorieva et al. [7] and Li and Liao [13] present recent advances in modelling of infectious diseases. Grigorieva and co-authors study the impact of non-linearity of functional responses (transmission terms in an SIR model) on the optimal control of infectious diseases. The authors apply the theory of optimal control and investigate the problem of optimisation of the number of infected individuals at a fixed time. As control measures, they consider various vaccination policies including vaccination, isolation of the infected and reduction of disease transmission. They prove that the optimal controls involves "bang-bang functions" and then estimate the maximum possible number of switchings during the control. The results are found for an arbitrary parametrisation of the transmission term, which make them rather general. The work of Li and Liao [13] lies in the new research area - modelling dynamics of computer viruses. This emerging area is mathematically close to the 'classical' modelling of infectious diseases [21,27]. Li and Liao investigate the role of the quarantine in reducing the level of computer viruses in networks. They developed a new delayed epidemic model of computer virus with hybrid quarantine strategies. The model have been comprehensively studied and the direction of Hopf bifurcation has been addressed. The results of modelling predict that hybrid quarantine strategies would efficiently inhibit viral spread and suppress oscillations in the system.

Due to complexity of the underlying biological systems, realistic mathematical models can include a large number of equations. However, under certain conditions an initially complex model can be transformed into a simpler one without losing the essential system properties by using model reduction 
techniques. For instance, complex spatially explicit systems can be sometimes reduced to mean-field models (see [16] for an introductory review). Bravo de la Parra et al. [4] consider the aggregation of variables method for the reduction of discrete dynamical systems, in which the reduced model can be formulated in terms of only a few global variables. The suggested reduction method takes advantage of time scale separation by considering slow and fast processes, however, it is substantially different from similar methods developed for continuous systems (e.g. [3]). The authors investigate the dynamics of a semelparous age-structured population, where individuals are distributed across the spatial sites connected to each other: the dispersal process is considered to be fast as compared to the population dynamics. It is investigated how different dispersal strategies that organisms use can affect the patterns of the global population dynamics. Moussaoui and Auger [17], present a short review on models of population and community dynamics which incorporate individual behaviour. The change of tactics among individuals within the population is assumed to be a fast process as compared to demographics. As an application of the general theory, a stage-structured population with behavioral variability of individuals is considered, including hawk, dove and bully tactics. Using aggregation of variables methods, it is demonstrated that for a low cost of fighting, the proportion of hawks within the population should become large. This provides a nontrivial extension of earlier results by Petrovskii \& Blackshaw $[18,19]$ that extreme forms of aggression can be beneficial for the population, especially under harsh environmental conditions, resulting in a large proportion of aggressive individuals.

The final contribution by Perthame and Souganidis [20] investigates the evolution of the dispersal of populations, which is a fundamental question in evolutionary ecology $[9,22]$. The authors search for the optimal (evolutionarily stable) dispersal in a spatially structured population experiencing rare mutations. They prove that in the limit of vanishing mutations, the final outcome of evolution will correspond to the lowest dispersal rate. It is shown how to compute the speed of evolution towards the evolutionary stable distribution. The authors considered that mutations act on the continuous dispersal trait by means of the Laplacian operator. This is a different approach as compared to the standard concept of adaptive dynamics where each time a mutant is introduced in to the system and its fitness is further considered. Mathematically, the asymptotic analysis of the problem is nontrivial and requires different treatment for the space and the genetic trait variables. In particular, for the trait variable the treatment was based on WKB approximation and viscosity solutions, whereas for treatment of the spatial variable, the maximum principle and Sobolev embedding theorems were used.

\section{References}

[1] M.W. Adamson, A. Y. Morozov. When can we trust our model predictions? Unearthing structural sensitivity in biological systems. Proc. R. Soc. A., 469 (2012), 20120500.

[2] W. G. Alharbi, S. V. Petrovskii. The impact of fragmented habitat's size and shape on populations with Allee effect. Math. Mod. Nat. Phen., 11 (2016), 5-16.

[3] P. Auger, R. Bravo de la Parra, J.-C. Poggiale, E. Sanchez, L. Sanz. Aggregation methods in dynamical systems and applications in population and community dynamics. Phys. Life. Rev., 5 (2008), 79-105.

[4] R. Bravo de la Parra, M. Marva, F. Sansegundo Fast dispersal in semelparous populations. Math. Mod. Nat. Phen., 11 (2016), 121-135.

[5] H. I. Egilmez, A. Yu. Morozov. Tri-trophic plankton models revised: importance of space, food web structure and functional response parametrisation. Math. Mod. Nat. Phen., 11 (2016), 17-34.

[6] I. Hanski. Single-species spatial dynamics may contribute to long-term rarity and commonness. Ecology, 66 (1985) 335-343.

[7] E.V. Grigorieva, E.N. Khailov, A. Korobeinikov. Optimal control for a SIR epidemic model with nonlinear incidence rate. Math. Mod. Nat. Phen., 11 (2016), 90-105.

[8] J.Z. Farkas, A. Yu. Morozov, E.G. Arashkevich, A. Nikishina. Revisiting the stability of spatially heterogeneous predatorprey systems under eutrophication. Bull. Math. Biol., (2015), 77 1886-1908.

[9] V. Hutson, S. Martinez, K. Mischaikow, G. T. Vickers. The evolution of dispersal. J. Math. Biol., 47 (2003), 6, $483-517$.

[10] S.E. Kingsland. Modeling nature: Episodes in the history of population ecology. 2d ed. Chicago: Univ. of Chicago Press, 1995. 
[11] R. Levins. Some demographic and genetic consequences of environmental heterogeneity for bio- logical control. Bull. Entomol. Soc. Am., 15 (1969) 237-240.

[12] M.A. Lewis, S.V. Petrovskii, J. Potts. The Mathematics Behind Biological Invasions. Interdisciplinary Applied Mathematics, Vol. 44. Springer, 2016.

[13] C. Li, X. Liao. The impact of hybrid quarantine strategies and delay factor on viral prevalence in computer networks. Math. Mod. Nat. Phen., 11 (2016), 106-120.

[14] J. Maynard Smith. Models in Ecology. Cambridge Univ. Press, 1974.

[15] C. Morris, H. Lecar. Voltage oscillations in the barnacle giant muscle fiber. Biophys. J., 35 (1981), $193-213$.

[16] A. Yu. Morozov, J.C.Poggiale. From spatially explicit ecological models to mean-field dynamics: The state of the art and perspectives. Ecol. Compl., 10 (2012), 1-11.

[17] A. Moussaoui, P. Auger. Effects of fast Hawk-Dove-Bully game on the dynamics of a stage-structured population. Math. Mod. Nat. Phen., 11 (2016), 136-154.

[18] S.V. Petrovskii, R.P. Blackshaw, B.-L. Li. Persistence of structured populations with and without the Allee effect under adverse environmental conditions. Bull. Math. Biol., 70 (2008), 412-437.

[19] S.V. Petrovskii, R.P. Blackshaw. Behaviourally structured populations persist longer under harsh environmental conditions. Ecol. Lett. 6 (2003), 455-462.

[20] B. Perthame, P. E. Souganidis. Rare mutations limit of a steady state dispersal evolution model. Math. Mod. Nat. Phen., 11 (2016), 155-167.

[21] J. Ren, Y. Xu., J. Liu. Investigation of dynamics of a virus-antivirus model in complex network. Physica A, 421 (2015) 533-540.

[22] O. Ronce. How does it feel to be like a rolling stone? Ten questions about dispersal evolution. Annu. Rev. Ecol. Evol. Syst., (2007) 38:231-253.

[23] G.A. ten Broeke, G.A.K. van Voorn, B.W. Kooi, J. Molenaar. Detecting tipping points in ecological models with sensitivity analysis. Math. Mod. Nat. Phen., 11 (2016), 48-73.

[24] H. Seno Mathematical modelling of metapopulation dynamics: Revisiting its meaning. Math. Mod. Nat. Phen., 11 (2016), 35-47.

[25] I.Yu. Tyukin, A.N. Gorban, T.A. Tyukina, J. Mohammed Al-Ameri, Yu.A.Korablev Fast sampling of evolving systems with periodic trajectories. Math. Mod. Nat. Phen., 11 (2016), 74-89.

[26] I. Yu. Tyukin. Adaptation in Dynamical Systems. Cambridge Univ. Press, 2011.

[27] F. Wang, Y. Zhang, C. Wang, J. Ma, S.J. Moon. Stability analysis of a SEIQV epidemic model for rapid spreading worms. Comput. Secur., 29 (2010) 410-418. 